\title{
QCD Sum Rules for Heavy Flavors
}

\author{
V.M. Braun \\ Institut für Theoretische Physik, Universität Regensburg, D-93040 Regensburg, Germany \\ E-mail: 'Vladimir. Braun@physik.uni-regensburg. de'
}

ABSTRACT: I give a short summary of QCD sum rule results for hadrons involving a heavy quark, with emphasis on recent developments.

\section{Decay constants: $f_{B}$ etc.}

QCD sum rules for D-meson and B-meson decay constants were among the first applications of this approach [i]1] [i] Early estimates [2] have given $f_{B} \sim 100 \mathrm{MeV}$ and $m_{b} \sim 4.8 \mathrm{GeV}$. In 1983 Aliev and Eletsky [i3] carried out the first quantitative analysis incorporating radiative corrections and relativistic effects (power-like $1 / m_{b}$ corrections) with the result $f_{B}=130 \pm 30 \mathrm{MeV}$. This old analysis remains generally valid today and subsequent refinements concentrated on two issues.

First, it was noticed by several authors [in] that the QCD sum rule result for $f_{B}$ is strongly sensitive to the value of the $b$-quark mass. More precisely, the sensitivity is mainly to the difference $m_{B}-m_{b}$ rather than $m_{b}$ itself, but anyhow lowering $m_{b}$ by $200 \mathrm{MeV}$ produces an increase in $f_{B}$ by $\sim 50 \mathrm{MeV}$. Another way to formulate the same problem is that the QCD sum rule does not have enough accuracy to predict the values of $m_{b}$ and $f_{B}$ simultaneously. Hence, $m_{b}$ has to be taken from elsewhere as an input parameter.

Second, using the heavy quark effective the-

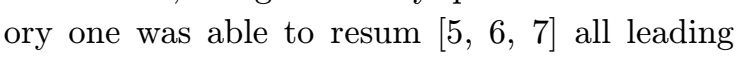
logarithmic contributions to the sum rule of the type $\left(\alpha_{s}\left(m_{b}\right) \ln m_{b} / \mu\right)^{k}$. The net effect of this resummation is that the strong coupling in the radiative correction has to be taken at a low scale of order $1 \mathrm{GeV}$ rather than $m_{b}$, as was assumed in [i․]. Combined with higher $\alpha_{s}$ values accepted nowadays, this resummation induces an increase in $f_{B}$ by $30-60 \mathrm{MeV}$, depending on further details.

The issue of the $b$-quark mass has recently been reexamined using new results [1] on the resummation of Coulombic corrections to heavyheavy correlation functions. Several independent studies (see Table. 1) all result in rather large quark mass values, not inconsistent with $m_{b}=$ 4.8 used in early QCD sum rule estimates. The possibility to pin down the $b$-quark mass with good precision is encouraging as it means that the sensitivity of sum rules on $m_{b}$ is a less serious problem as thought before. To match the accuracy of NNLO NRQCD calculations, one may try to calculate the $O\left(\alpha_{s}^{2}\right)$ corrections to the sum rule which is possible by existing methods. The BLM-type estimate [i rection is probably not large. One has to have in mind, however, that accuracy of QCD sum rules cannot be consistently improved by calculated higher order corrections and such calculations are only meaningful as to exclude (unexpected) large effects.

To summarize, I give my personal "weighted average"

$$
f_{B}=160 \pm 30 \mathrm{MeV}
$$

which is in agreement with other estimates [1]", [19.", is stable for may years and, unfortunately, not improvable. Note that this value is consistent with the old result of Aliev-Eletsky from 1983 and the error is actually not reduced despite significant effort that had been invested ever since.

The coupling $f_{D}$ historically attracted less attention. Aliev and Eletsky [3] have obtained $f_{D}=170 \mathrm{MeV}$ with claimed accuracy of order $20 \%$. The dependence on $c$-quark mass happens to be somewhat milder in this case. The recent 


\begin{tabular}{|c|c|c|c|}
\hline & $m_{b}$ & $\bar{m}_{b}\left(\bar{m}_{b}\right)$ & Remarks \\
\hline BS99 [1] [1] & $4.97 \pm 0.17$ & $4.25 \pm 0.08$ & Sum rules \\
\hline PP98 [1] & $4.80 \pm 0.06$ & - & Sum rules \\
\hline H98 [1] 2] & $4.88 \pm 0.10$ & $4.20 \pm 0.06$ & Sum rules \\
\hline MY98 [1] & - & $4.20 \pm 0.10$ & Sum rules \\
\hline JP97/98 [1] & $4.60 \pm 0.02$ & $4.19 \pm 0.06$ & Sum rules, no resummation \\
\hline PY98 [1] $\left[\begin{array}{l}1 \\
2\end{array}\right]$ & $5.00_{-0.07}^{+0.10}$ & $4.44_{-0.04}^{+0.03}$ & $\Upsilon(1 \mathrm{~S})$ mass \\
\hline GGRM99 [1] & - & $4.26 \pm 0.08$ & lattice HQET \\
\hline
\end{tabular}

Table 1: Summary of recent determinations of the $b$-quark mass. The table is adapted from [9in] and updated

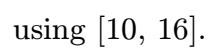

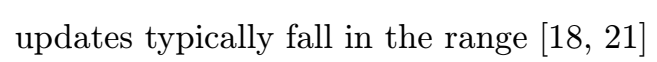

$$
f_{D}=190 \pm 30 \mathrm{MeV}
$$

where the small increase is mainly due to a higher value of $\alpha_{s}$.

Calculation of $S U(3)$-breaking effects in the QCD sum rules is not easy because several effects tend to compensate each other. This difficulty is reflected in the rather large errors

$$
\begin{aligned}
& f_{D_{s}} / f_{D}=1.19 \pm 0.08, \\
& f_{B_{s}} / f_{B}=1.19 \pm 0.08 .
\end{aligned}
$$

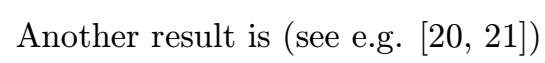

$$
\begin{aligned}
& f_{D^{*}} / f_{D}=1.40 \pm 0.15, \\
& f_{B^{*}} / f_{B}=1.10 \pm 0.08 .
\end{aligned}
$$

Finally, with the advent of the Heavy Quark Effective Theory (HQET) it has become customary to present relativistic corrections [in] to $f_{B}$ in the form of a series expansion in powers of the heavy quark mass:

$$
f_{B}=\widehat{C}\left(m_{b}\right) F_{\text {stat }}\left[1-\frac{A}{M_{b}}+\ldots\right] .
$$

Here $F_{\text {stat }}$ is a universal nonperturbative constant, $\widehat{C}\left(m_{b}\right)$ can be calculated perturbatively and the power corrections can be related to matrix elements of higher dimension HQET operators. The QCD sum rules give very stable pre-

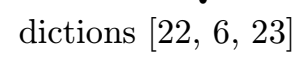

$$
A=0.9 \pm 0.2 \mathrm{GeV} .
$$

\section{HQET parameters $\bar{\Lambda}, \lambda_{1}, \lambda_{2}$}

QCD sum rules have a well-defined heavy quark limit and can be used to estimate HQET parameters, see [2]4] for the relevant definitions. For example, one obtains for the difference between meson and quark masses

$$
\bar{\Lambda} \simeq 400-500 \mathrm{MeV} .
$$

This value is consistent with $m_{b}$ determinations although the accuracy is not competitive. More interestingly, the sum rule for the chromomagnetic interaction parameter $\lambda_{2}$ comes out to be rather stable. Translated into the prediction for the mass splitting between vector and pseudosca-

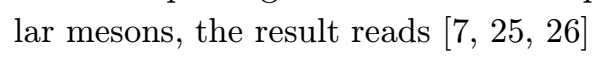

$$
\left(m_{V}^{2}-m_{P}^{2}\right)^{\mu=m_{b}}=(0.46 \pm 0.14) \mathrm{GeV}^{2},
$$

which compares very well with the experimental value $m_{B^{*}}^{2}-m_{B}^{2} \simeq 0.48 \mathrm{GeV}^{2}$. A technical remark is that all sum rule results in HQET apear to be strongly correlated with the value of $\bar{\Lambda}$ which is just another manifestation of the sensitivity of "ordinary" sum rule on the value of $m_{b}$. To reduce this dependence, and also to moderate radiative corrections, one often attempts to consider ratios of sum rules in which such effects tend to cancel.

The most interesting quantity is $-\lambda_{1}$, the $b$ quark kinetic energy in the B-meson. Knowledge of $\lambda_{1}$ is crucial for the accuracy of $V_{c b}$ determinations from inclusive B-decays using OPE. The situation with $\lambda_{1}$ determination from QCD sum rules was controversial for some time, with two calculations reporting conflicting results: $-\lambda_{1}=$ 
$0.5 \pm 0.2 \mathrm{GeV}^{2}[25]$ and $-\lambda_{1}=0.1 \pm 0.05 \mathrm{GeV}^{2}$ $\left[26_{1}^{\prime}\right]$. By now, the origin of this discrepancy is well understood [2] $\overline{7}_{1}$, but the remedy is not found. The difficulty can be traced to contributions of nondiagonal matrix elements $\sim\left\langle B\left|\bar{h}\left(D_{\perp}^{2}\right) h\right| B^{\prime}\right\rangle$ where $B^{\prime}$ is a certain excited pseudoscalar meson state, which are difficult to disentangle from the "diagonal" contribution of interest within the QCD sum rule framework. Several toy-model calculations using quantum-mechanical examples [28'] strongly suggest that such contributions are present in both sum rules [25] and [2 $\left.2 \overline{6}_{1}^{\prime}\right]$ and have opposite signs. In quantum mechanics it is always possible to add the two sum rules analogous to $\left[25_{-1}, \overline{2}_{6}\right]$ with some weight (fixed by the virial theorem) such that the nondiagonal transitions cancel exactly. In QCD the corresponding weight is not known. Therefore, for the time being I prefer to take the results of $\left[2 \overline{2}^{1}, \overline{2}, \overline{2} \overline{6}_{1}^{\prime}\right]$ as providing for the upper and the lower boundary, respectively, leading to a conservative average

$$
-\lambda_{1}=0.35 \pm 0.20 \mathrm{GeV}^{2} .
$$

Doing better is an open problem. An unrelated difficulty is that the value for $-\lambda_{1}$ may depend rather strongly on its precise definition, in particular whether or not a Lorentz-invariant UV cutoff is used.

\section{3. $B \rightarrow D, \ldots$ form factors and Isgur- Wise functions}

In the period 1989 - 1992 several sum rules have been derived and studied for the semileptonic decays of beauty to charm, using the standard QCD sum rule maschinery and finite values for heavy quark masses $[29-1,130,1]$. In later works the accent has shifted to incorporate the heavy quark expansion and calculate the Isgur-Wise functions instead of the form factors themselves. Such approach has an advantage that the heavy quark symmetry is incorporated analytically rather than numerically, but at the same time raises concerns on validity of the $1 / m_{c}$ expansion.

A lot of effort went into calculation of the celebrated Isgur-Wise function $\xi(y)$ and its slope $\rho=\xi^{\prime}(y=1)$ as providing main input for the $V_{c b}$ determinations. The leading-order sum rules

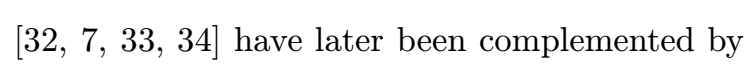
calculation of the radiative corrections [35in. The result is

$$
\rho=0.7 \pm 0.25
$$

and probably cannot be improved. The discussion around this sum rule was actually beneficial for the general development of the sum rule approach, allowing to clarify certain important points about continuum subtraction, see [3 $\left.3 \overline{4}_{i}^{1}\right]$ for details.

QCD sum rules have also been derived for the Isgur-Wise functions $\tau_{1 / 2}(y)$ and $\tau_{3 / 2}$ which govern semileptonic transitions to positive parity states $\frac{1}{2}^{-} \rightarrow \frac{1}{2}^{+}, \frac{3}{2}^{+}$and contribute e.g. to the Bjorken inequality

$$
\rho>\frac{1}{4}+\left|\tau_{1 / 2}(1)\right|^{2}+\left|\tau_{3 / 2}(1)\right|^{2} .
$$

The sum rule for $\tau_{3 / 2}$ is only available to leading order [ [3 $\left.\overline{6}_{1}\right]$ while for $\tau_{1 / 2}$ the radiative corrections

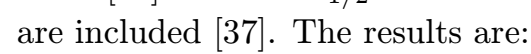

$$
\begin{gathered}
\tau_{1 / 2}(1)=0.35 \pm 0.08, \rho_{1 / 2}^{2}=2.5 \pm 1.0\left[\overline{3} \overline{3} \overline{7_{1}}\right] \\
\tau_{3 / 2}(1) \sim 0.3, \rho_{3 / 2}^{2} \sim 0.9[\overline{3} \overline{6} \overline{1} .
\end{gathered}
$$

For illustration of the accuracy, I show one typical plot:

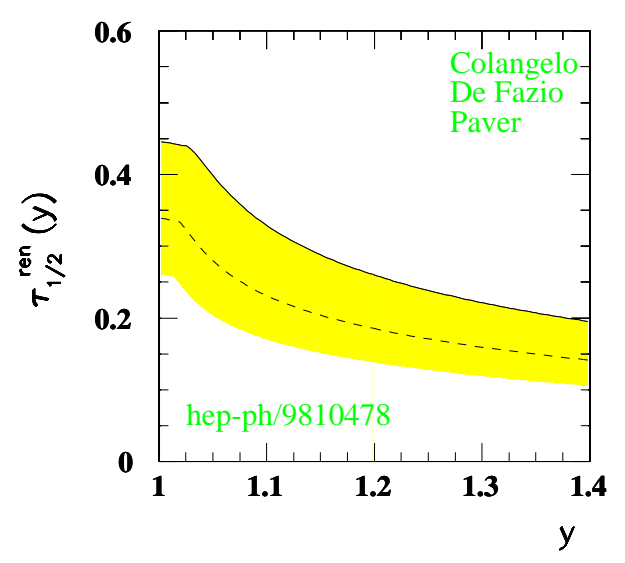

Figure 1: The Isgur-Wise function $\tau_{1 / 2}$. Figure taken from [3]

Finally, certain results are available for subleading Isgur-Wise functions $\chi_{1,2,3}(y)$ and $\xi_{3}(y)$

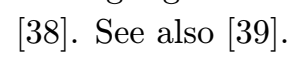

\section{Semileptonic D decays}

Calculations of the form factor values at $q^{2}=0$ have a long history [40]-[4] 
old. The results are [4]

$$
\begin{aligned}
f_{+}^{D \rightarrow \pi}(0) & =0.50 \pm 0.15 \\
f_{+}^{D \rightarrow K}(0) & =0.60 \pm 0.15
\end{aligned}
$$

and

$$
\begin{aligned}
A_{1}^{\rho}(0) & =0.50 \pm 0.2, \\
A_{1}^{K^{*}}(0) & =0.50 \pm 0.15, \\
A_{2}^{\rho}(0) & =0.40 \pm 0.1, \\
A_{2}^{K^{*}}(0) & =0.60 \pm 0.15, \\
V^{\rho}(0) & =1.0 \pm 0.2, \\
V^{K^{*}}(0) & =1.1 \pm 0.25 .
\end{aligned}
$$

The quoted values for $f_{+}^{D \rightarrow \pi}(0)$ and $f_{+}^{D \rightarrow K}(0)$ are somewhat too low, to my opinion, and result from using a too small value for the quark condensate. The other entries are less affected by this choice.

QCD sum rule predictions for the $q^{2}$ dependence of the form factors [43] have been the most interesting. While the shape of the form factors $f_{+}$and $V$ turned out to be consistent with vector dominance, the axial-vector form factors $A_{1}$ and $A_{2}$ came out almost flat. Such behaviour was unexpected and initiated a lively discussion which continues nowadays. It is worth while to mention that sum rule calculations of the form factors in the physical region $q^{2}>0$ are technically nontrivial because of subtleties in the construction of double dispersion relations in presence of Landau thresholds, see [4흔] for the details.

In addition, the form factor $f_{+}^{D \rightarrow \pi}\left(q^{2}\right)$ was analysed using light-cone sum rules (LCSR) in $\left[20^{\prime}\right]$ (see the next section for a detailed description of this approach). Very recently, the NLO corrections to this sum rule have been calculated

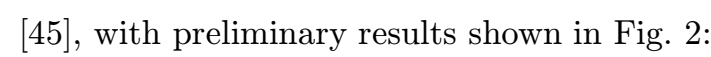

The LCSR result is shown by the solid curve. It is valid at small $q^{2}<1 \mathrm{GeV}^{2}$ and is matched at higher $q^{2}$ to the vector dominance prediction using a yet another new LCSR estimate [2]

$$
f_{D^{*}} g_{D D^{*} \pi}=2.84 \pm 0.6 \mathrm{GeV}^{2} .
$$

In [4듬] the ratio of decay widths $D \rightarrow K$ and $D \rightarrow \pi$ is calculated and claimed to be strongly sensitive to the value of the strange quark mass:

$$
f_{+}^{D \rightarrow \pi}(0)=0.66 \pm 0.09
$$

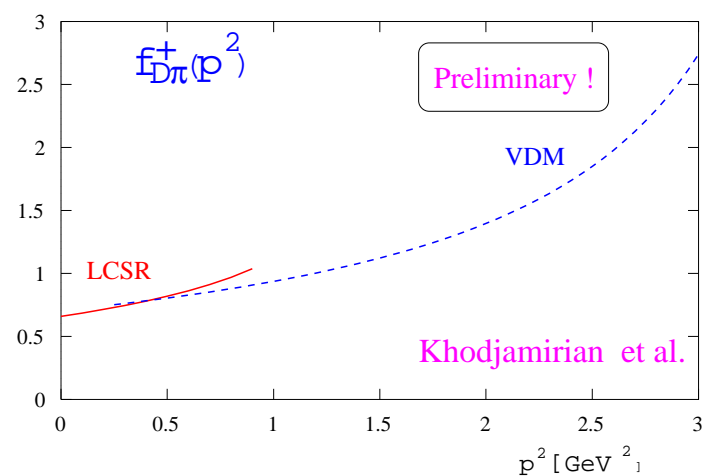

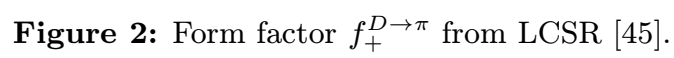

$$
f_{+}^{D \rightarrow K}(0)= \begin{cases}0.98 \pm 0.11, & m_{s}=100 \mathrm{MeV} \\ 0.77 \pm 0.11, & m_{s}=150 \mathrm{MeV} \\ 0.66 \pm 0.09, & m_{s}=200 \mathrm{MeV}\end{cases}
$$

The scalar form factor $f_{0}^{D \rightarrow \pi}$ was considered in [46] using LCSR. At $q^{2}=0$ the result is

$$
\left.f_{0}^{D \rightarrow \pi}(0)=f_{+}^{D \rightarrow \pi}(0)=0.68 \text { [i } \underline{4}^{-}{ }_{-}\right]
$$

with, probably, $20 \%$ error.

\section{Semileptonic and rare $B$ decays}

These decays typically involve a large momentum transfer to the light hadron in the final state and have to be treated with care. The corresponding technique, known as light-cone sum rules, was

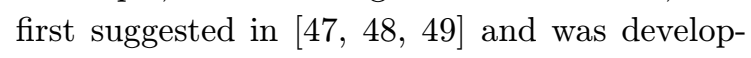
ing rapidly during past few years. In essence, it presents a generalization of the classical meanfield type SVZ approach to expansion of correlation functions in rapidly varying (large momentum) background meson fields. Technically, the difference is that the short-distance expansion in operators of increasing dimension is replaced by the light-cone expansion in increasing twist. On this way, vacuum averages of quark and gluon fields (condensates) do not appear and are replaced by light-cone meson distribution amplitudes, see [501, 51, for the two recent expositions. Premium for this rearrangement is that the approach becomes explicitly consistent with the heavy quark expansion. A detailed comparison of light-cone and conventional QCD sum rules can be found in [52"].

The decay $B \rightarrow \pi e \nu$ was considered many times by different authors using both conven- 


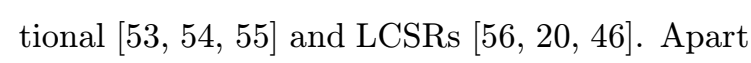
from phenomenological importance, this decay serves as a convenient test ground for various versions of sum rules and other theoretical ideas. The new results which I want to report on this meeting, are obtained using LCSR calculations with NLO accuracy, including radiative correc-

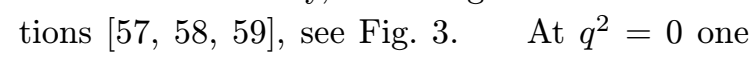

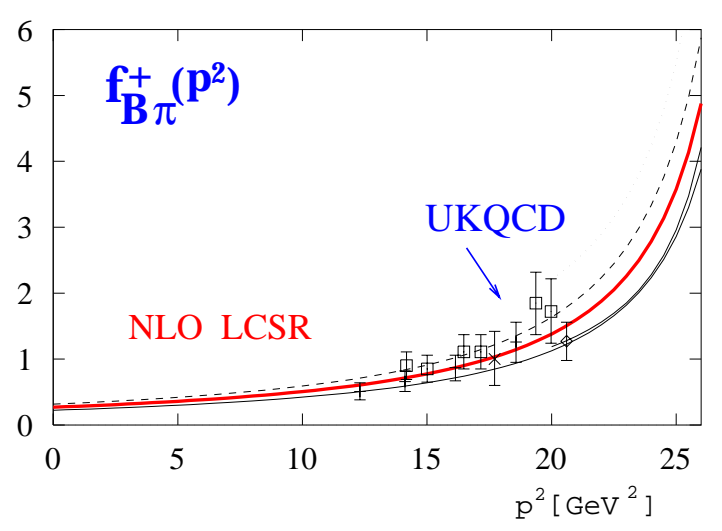

Figure 3: Form factor $f_{+}^{B \rightarrow \pi}$ from LCSR [4-

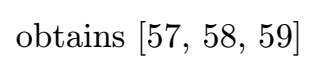

$$
f_{+}^{B \rightarrow \pi}=0.27 \pm 0.05
$$

where the largest uncertainty comes from pion distribution amplitude. The calculation automatically satisfies the unitarity constraints [60 as illustrated in Fig. 迹. It should be mentioned that the LCSR approach is only justified sufficiently far from zero recoil, as indicated by arrows in in Fig. 'í,', and in this region cannot violate any unitarity bounds. My personal opinion is that unitarity constraints are not sufficiently restrictive and for small $q^{2}$ do not provide any additional knowledge (for heavy-to-light decays) compared to "reasonable" model calculations.

Many new results were obtained recently for B-decays into light vector mesons. Early LCSR

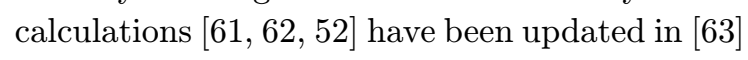
by taking into account radiative corrections, meson mass corrections and contributions of meson wave functions of higher twist [64in].

The results are presented in Fig. particular case $B \rightarrow K^{*}$. Note the error bands and good agreement with lattice calculations by the UKQCD collaboration [66]'. Several simple parametrizations of the $q^{2}$-dependence are available, see e.g. $[63$
Unitarity Bounds: Boyd, Rothstein '97

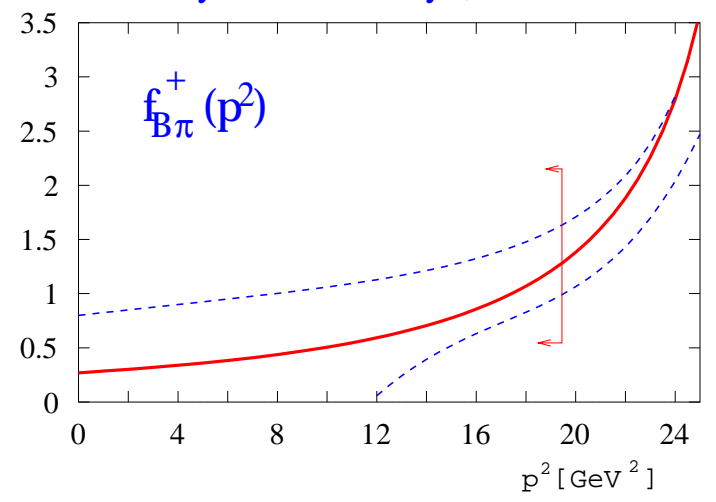

Figure 4: Form factor $f_{+}^{B \rightarrow \pi}$ from LCSR (solid curve) compared with the unitarity bounds (dashes). Figure taken from [푸해].

at $q^{2}=0$ are compiled in Table 2. Note general agreement between different LCSR calculations and also with lattice results. For a detailed analysis of the phenomenological implications of these results see [6흐.

The b-quark mass dependence of the form factors deserves a special discussion. As observed in $[4 \overline{9}, 1, \overline{1}, 1]$ all form factors in question have a universal behaviour $\sim 1 / \mathrm{m}_{b}^{3 / 2}$ in the heavy quark limit. This scaling law is suggested by the analysis of leading integration regions in Feynman diagrams (see discussion in $[5,5,50$ ) and is valid for both "soft" and "hard" contributions separately. To be precise, the scaling is $\sim \sqrt{m_{b}} / E^{2}$

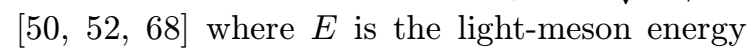
in the final state (in the B-meson rest frame). The factor $1 / E^{2}$ arises invariantly either as gluon virtuality in hard rescattering, or from the overlap integral between soft wave functions, while $\sqrt{m_{b}}$ comes from the B-meson wave function normalization. This scaling law is supported by LC sum rules which also suggest that the soft contribution is dominant for realistic b-quark mass values. If the hard rescattering correction is neglected, several symmetry relations can be derived between semileptonic and rare decay form factors in the heavy quark limit, see $[6 \overline{8} \overline{0}]$. The LCSR calculations are explicitly consistent with these relations and in fact suggest that the corrections are numerically rather small $[\overline{6} 6 \overline{1} 1, \overline{6}, \overline{3} 3, \overline{1}, \overline{6} \overline{8}]$. On the other hand, the b-quark mass dependence comes out to be rather far from the asymptotic 

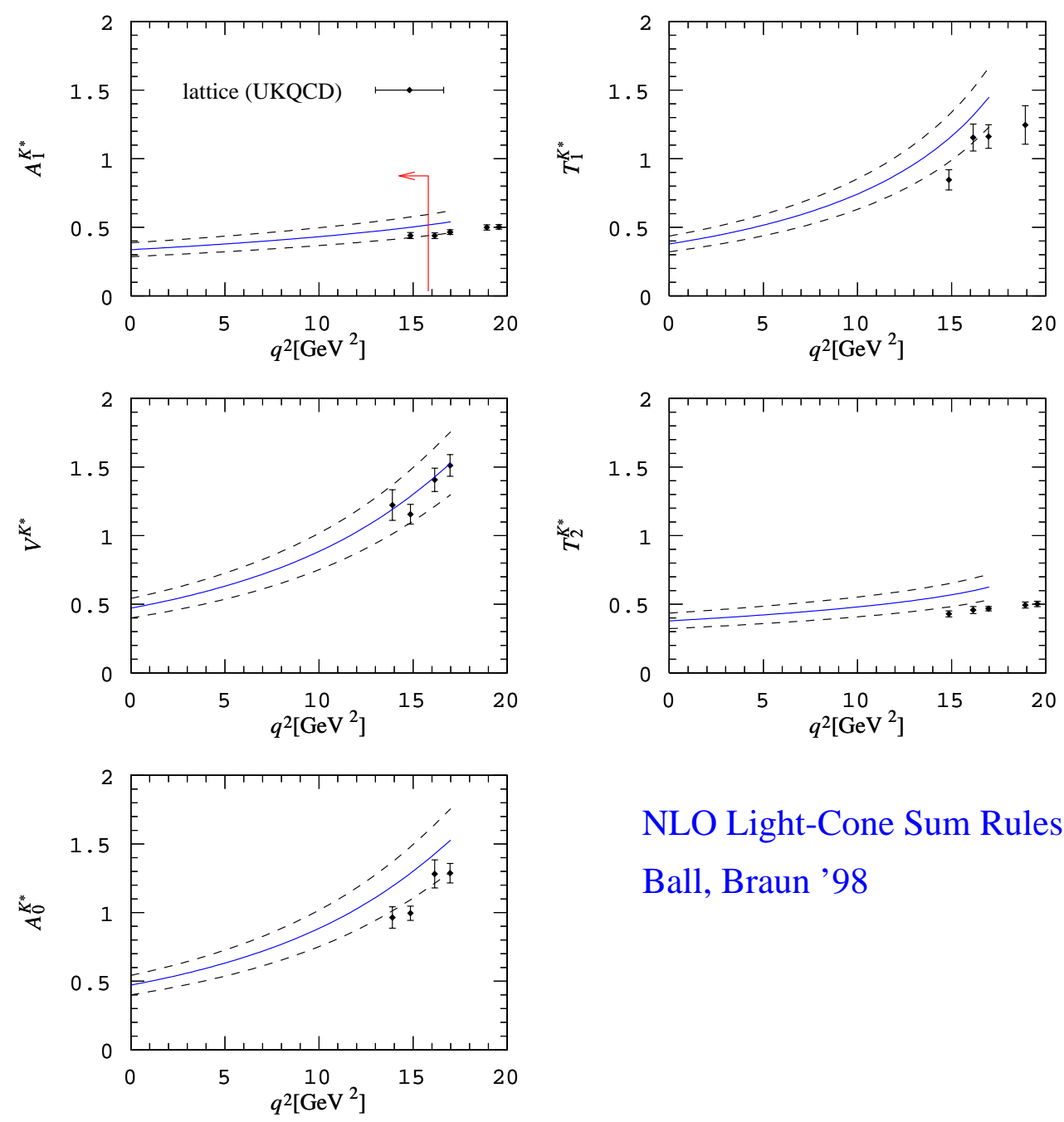

\section{NLO Light-Cone Sum Rules}

Ball, Braun '98

Figure 5: Rare decay $B \rightarrow K^{*}$ form factors from LCSR [63]

scaling limit. Rough estimates $\left[\overline{6}_{1} \overline{1}_{-}^{1}, \overline{4} \overline{6}_{1}\right]$ suggest large preasymptotic corrections of order

$$
F\left(q^{2}=0\right) \sim m_{b}^{-3 / 2} F_{\text {stat }}\left[1-\frac{C}{m_{b}}+\ldots\right]
$$

with $C \sim 1-1.5 \mathrm{GeV}$.

As far as technical implementation is concerned, the existing LCSR calculations present the state of the art of this approach and it will be difficult to improve them in near future. They can be updated if new information about lightcone meson distribution functions becomes available. There exist, however, a few issues which still have to be clarified. First, LC sum rules systematically indicate larger $\mathrm{SU}(3)$ breaking corrections compared to lattice calculations, and the origin of this is not understood. Second, in the treatment of sum rules themselves one probably can improve on the treatment of kinematical factors (separation of Lorentz structures and invariant amplitudes), in particular, going over to calculations of helicity amplitudes.

\section{6. $g_{B^{*} B \pi}, g_{D^{*} D \pi}$ etc.}

A detailed study of the pion coupling to heavy mesons was carried out in [20 cone and conventional sum rules with the result

$$
\begin{aligned}
& g_{B^{*} B \pi}=29 \pm 3 \\
& g_{B^{*} B \pi}=12.5 \pm 1,
\end{aligned}
$$

where the errors are probably underestimated. 


\begin{tabular}{|c|c|c|c|c|}
\hline & $\begin{array}{c}\text { BB98 [6]" } \\
\text { (NLO LCSR) }\end{array}$ & $\operatorname{ABS}\left[\begin{array}{c}61 \\
\text { (LCSR) }\end{array}\right.$ & $\begin{array}{l}\text { AOS [6\%"] } \\
\text { (LCSR) }\end{array}$ & $\begin{array}{c}\text { UKQCD [6 } 6 \overline{6}] \\
\text { (Lattice + LCSR) }\end{array}$ \\
\hline$A_{1}^{\rho}(0)$ & $0.26 \pm 0.04$ & $0.27 \pm 0.05$ & $0.30 \pm 0.05$ & $0.27_{-0.04}^{+0.05}$ \\
\hline$A_{2}^{\rho}(0)$ & $0.22 \pm 0.03$ & $0.28 \pm 0.05$ & $0.33 \pm 0.05$ & $0.26_{-0.03}^{+0.05}$ \\
\hline$V^{\rho}(0)$ & $0.34 \pm 0.05$ & $0.35 \pm 0.07$ & $0.37 \pm 0.07$ & $0.35_{-0.05}^{+0.06}$ \\
\hline$T_{1}^{\rho}(0)$ & $0.29 \pm 0.04$ & $0.24 \pm 0.07$ & $0.30 \pm 0.10$ & - \\
\hline$T_{3}^{\rho}(0)$ & $0.20 \pm 0.03$ & - & $0.20 \pm 0.10$ & - \\
\hline$A_{1}^{K^{*}}(0)$ & $0.34 \pm 0.05$ & $0.32 \pm 0.06$ & $0.36 \pm 0.05$ & $0.29_{-0.03}^{+0.04}$ \\
\hline$A_{2}^{K^{*}}(0)$ & $0.28 \pm 0.04$ & - & $0.40 \pm 0.05$ & - \\
\hline$V^{K^{*}}(0)$ & $0.46 \pm 0.07$ & $0.38 \pm 0.08$ & $0.45 \pm 0.08$ & - \\
\hline$T_{1}^{K^{*}}(0)$ & $0.38 \pm 0.06$ & $0.32 \pm 0.05$ & $0.34 \pm 0.10$ & $0.32_{-0.02}^{+0.04}$ \\
\hline$T_{3}^{K^{*}}(0)$ & $0.26 \pm 0.04$ & - & $0.26 \pm 0.10$ & - \\
\hline
\end{tabular}

Table 2: Comparison of results from different works on $B \rightarrow \rho, K^{*}$ form factors at $q^{2}=0$ [6 $\left.63_{1}^{1}\right]$.

In the heavy quark limit one obtains "[20̄"

$$
\begin{aligned}
g_{B^{*} B \pi} & =\frac{2 m_{B}}{f_{\pi}} \hat{g}\left(1+\frac{\Delta}{m_{B}}\right), \\
\hat{g} & =0.32 \pm 0.02 \\
\Delta & =(0.7 \pm 0.1) \mathrm{GeV} .
\end{aligned}
$$

Very recently, radiative corrections to these sum rules have been calculated [21 1 . They turn out to be negative and decrease the values of the couplings by approximately $20 \%$. To my opinion this reduction has to be taken with some caution since the main input parameter in this sum rule - the pion distribution amplitude in the middle point - was determined in [4욜] without taking radiative corrections into account. The analysis of [4을 in in fact quite old and its update is long overdue.

Similar sum rules (to leading order only) were derived for decay constants $g_{B^{*} B \rho}$ and $g_{D^{*} D \rho}$

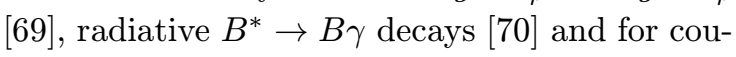
plings to positive parity heavy mesons [7]1', $\left.77_{2}^{2}\right]$.

\section{Other topics in B-decays}

Concluding this part, I want to mention two other applications of sum rules which I find interesting.

First, an exploratory study of nonfactorizable contributions to the $B \rightarrow J / \Psi K$ decay $[51$, 73in. In the usual factorisation approximation the corresponding matrix element is proportional to the sum $a_{2}=c_{2}\left(m_{b}\right)+\frac{1}{3} c_{1}\left(m_{b}\right) \simeq 0.155$, where $c_{1}$ and $c_{2}$ are the coefficients in the effective Lagrangian in common notation. The experimental data are usually analysed introducing an effective coefficient $a_{2, \text { eff }}^{B \psi K}$ to take into account nonfactorizable effects and the current result $\left|a_{2, \exp }^{B \psi K}\right|=0.31 \pm 0.02$ can be interpreted as strong violation of the factorization. The effective coefficient $a_{2}$ was estimated in QCD sum

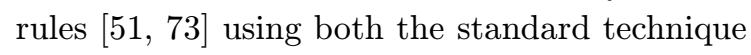
[747] developed originally for D-decays, and the LCSRs. Numerical results are still very unstable and preliminary, but, interestingly enough, the sum rules seem to indicate a negative value for $a_{2}$. Although calculations are difficult, potentially this is a large and interesting field of applications.

Second, the LCSR approach can be and was already used to estimate long-distance contributions in decays like $B \rightarrow K^{*} \gamma, B \rightarrow \rho^{*} \gamma$, see [775,

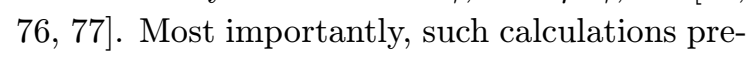
dict the relative sign of short- and long-distance amplitudes and, therefore, their interference. The existing results can be improved and extended to $B \rightarrow K^{*} \ell \bar{\ell}$ and similar transitions. The sum rules of this type are rather well understood and can be expected to give reliable predictions.

\section{Heavy baryons}

Studies of heavy baryons in QCD sum rules are 
in general difficult. They have been going on for some time, but, to my opinion, the results still are rather preliminary. I will discuss two applications to problems of direct phenomenological relevance.

First is the calculation of baryon matrix elements of four-fermion operators [7] responsible for the $1 / m_{b}^{2}$ corrections to life-time differences between beautiful baryons and mesons. The corresponding sum rules are very unstable because of high dimension and can only produce an order of magnitude estimate. The result of the calculation is negative: Despite all uncertainties one can claim that the relevant matrix elements cannot be as large as to explain the observed ratio $\tau\left(\Lambda_{d}\right) / \tau\left(B_{d}\right)$.

Second, several QCD sum rule calculations exist of the semileptonic decays of heavy baryons $\Lambda_{b} \rightarrow \Lambda_{c} \ell \bar{\nu}, \Lambda_{c} \rightarrow \Lambda \ell \bar{\nu}$ and $\Lambda_{b} \rightarrow p \ell \bar{\nu},\left[\overline{7} \bar{g}_{1}^{\prime}, \overline{8} \overline{0}_{1}^{\prime}\right.$ 181'], see Fig. ' $\overline{6}_{p}^{\prime}$ and also of rare decays $\Lambda_{b} \rightarrow \Lambda \bar{\gamma}$, $\Lambda_{b} \rightarrow \Lambda \ell \bar{\ell}\left[8{ }^{\prime}\right] . \quad$ For the decay widths one

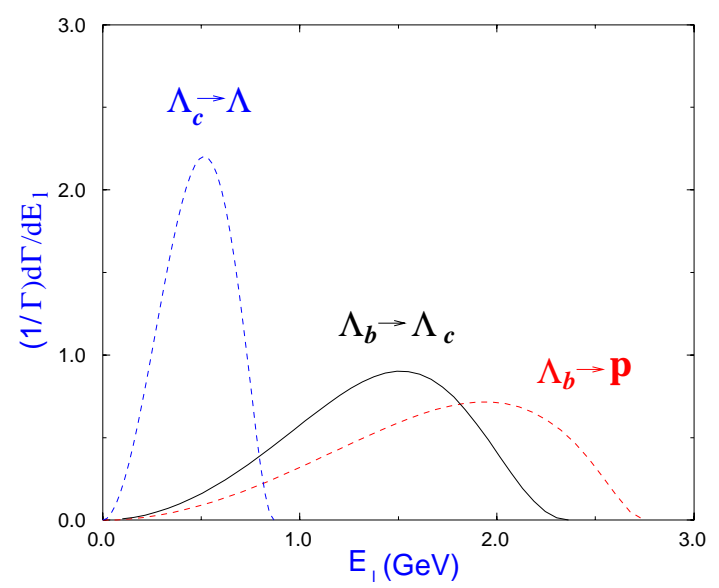

Figure 6: Lepton energy spectra in decays of heavy baryons [in].

obtains, e.g. [8 $\left[8 i^{\prime}\right]$

$$
\Gamma\left(\Lambda_{b} \rightarrow p \ell^{-} \bar{\nu}_{\ell}\right)=(1.7 \pm 0.7) \times 10^{-11}\left|V_{u b}\right|^{2} \mathrm{GeV}
$$

All these studies are carried out using traditional three-point sum rules. The LCSR technology has not been applied yet because of almost complete absence of information on higher-twist baryon wave functions. In the case of a light baryon in the final state, the same critisism applies, therefore, to this calculations as for heavy-to-light meson transitions [52].
Baryonic Isgur-Wise functions have been considered in [83 topic and other applications.

\section{Conclusions}

The main new development in the QCD sum rules during the past couple of years have been, to my opinion, the arrival of a new generation of NLO LCSR calculations, incorporating radiative corrections and new results on meson wave functions of leading and higher twists. Detailed state-of-the-art studies are now available using such sum rules for the form factors of heavy-tolight transitions at large recoil.

Among the problems where further developments can be expected and the sum rules are likely to make an impact, I can mention:

- Light-cone sum rules for heavy baryons. Dedicated studies of higher-twist baryon distribution amplitudes beyond leading twist are, however, required as a first step.

- Long-distance contributions to various rare decays.

- Nonfactorizable contributions to nonleptonic decays like $B \rightarrow \pi \pi$ etc.

- Matrix elements of penguin operators.

Interesting problems where exploratory studies are neccessary to determine feasibility of the QCD sum rule calculations are, among others: Colouroctet matrix elements in charmonium, parton distributions in B mesons and $B_{c}$ decays (see Further progress can eventually be achieved in cooperation of sum rules with lattice calculations (e.g. to determine meson distribution amplitudes) and by studying exclusive processes with light hadrons at comparable momentum transfers.

\section{Acknowledgments}

I am grateful to A. Ali, E. Bagan, I. Balitsky, P. Ball, H.G. Dosch, I. Halperin, A. Khodjamirian, R. Rückl and H. Simma for collaboration on the topics covered in this report. Special thanks are due to A. Khodjamirian and R. Rückl for the possibility to use the results of [4 450 prior publication. 


\section{References}

201 V.M. Belyaev et al., PPhys. Rev. D. 51 (1995)

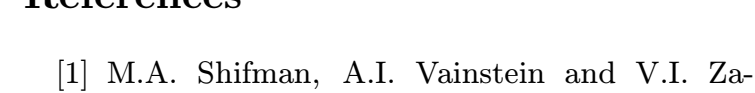

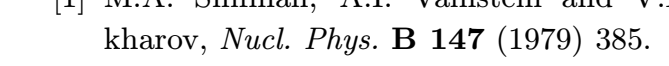
6177

-

[21] A. Khodjamirian et al., 'Phys. Lett. B (1999) 245

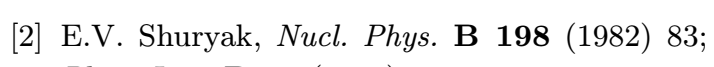

- - - Physs: Eett.-B-93-(-1980)-134:-

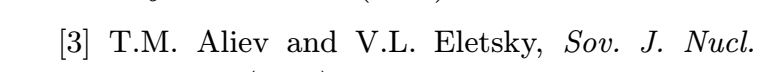

Phys $38(1983) 936$

[4] C.A. Dominguez and N. Paver, 'Physs. Lett. B' ;

- - - L.J. Reinders

S. Narison, 'Phys. Lett. B 198 (1987)_104'; P. Colangelo et al., 'Phys. Lett. B 269 (1991)' :

$22 \_$V. Eletsky and E. Shuryak, 'Phys. Lett. B_276' $(1992) 191 !$

[5] D.J. Broadhurst and A.G. Grozin, Physs. Lett.' $\mathrm{i}=-\mathrm{B}-274-(1992)-42 \overline{1}$

[6] E. Bagan et al., Phys. Lett. B_278_(1992) 457.

; $[\overline{7}] \mathrm{M}$. Neubert, PPhys. Rev. D-45 (1992) 2451.

[23] P. Ball, 'Nucl. Phys. B $4 \mathbf{2} 1$ (1994) 593

[24] M. Neubert, iPhys. Rep. 245 (1994) $259_{1}$

[25]_P. Ball and V.M. Braun 'Phys. Rev. D _49 $(1994) 2472$

[26] M. Neubert, 'Phys. Lett. B $\mathbf{3 8 9}(1996) 72 \bar{T}_{1}$

[27] I. Bigi, M. Shifman and N. Uraltsev, Ann. Rev.' Nucl. Part. Sci. 47 (1997) 591.

[28] B. Blok and M. Lublinsky, 'Physs. Rev. D. $\mathbf{5} \bar{z}_{1}^{\prime}$ (1998) 2676; ibid. 58 (1998) $019903(\mathrm{E})$.

[8] M. Beneke, these proceedings.

[29] A.A. Ovchinnikov and V.A. Slobodenyuk, 'S- Sov.: J. Nucl. Phys. 50 (1989) 891 .

L - - [9]- ML. -Beneke, -A.- Signer- art- -V-

[30L V.N. Baier and A.G. Grozin, 'Z. Physsik C $\mathbf{4} \overline{z_{1}}$ (1990) 669:

Two loop application of the threshold expan-

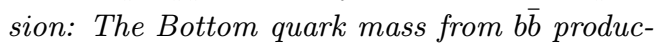
tion, hep-ph/9906476.

_10] M. Beneke and A. Signer, The Bottom MS-bar quark mass from sum rules at next-to-next-toleading order, hep-ph/9906475.

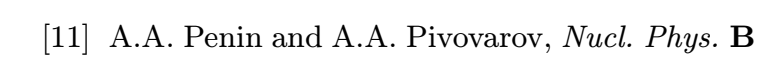
L-

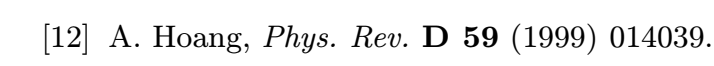

[31]_P. Colangelo et al., 'P 201.

[32] A.V. Radyushkin, 'Phys. Lett. B $\mathbf{2} 71$ 218

[33] P. Ball, 'Phys. Lett. B-281 (1992) 133.'.

[34] B. Blok and M. Shifman, Phys. Rev. D 47i $(1993) 2949$

[35] E. Bagan, P. Ball and P. Gosdzinsky, Phy Lett. B $301-1993) 249$

[13] K. Melnikov and A. Yelkhovsky, 1 h hys. Rev. Di M. Neubert, Phys. Rev. D_ 47 (1993) 4063.

: 59 (1999) 114009'.

_14]_M Jaminand_A Pich (1997) 334 inep-ph/9810259

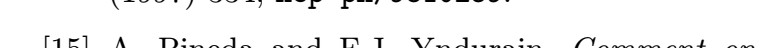

_. _15]_ A. Pineda_and_ F.J. _Yndurain, Comment _on 'Calculation of quarkonium spectrum and $m_{b}$, $m_{c}$ to order $\alpha_{s}^{4}$, hep-ph/9812371:

[36] P. Colangelo, G. Nardulli and N. Paver, P.Phys.' Lett. B 293 (1992) 207

[37] P. Colangelo, F. De Fazio and N. Paver, Phhys.' Rev. D $58-(1998) 116005$ hep-ph/9810478.

[38] M. Neubert, Phys. Rev. $\mathbf{4} \mathbf{6}(1992) 3914 ;$ M. Neubert, Z. Ligeti and Y $\mathrm{Nir}$, Phys. Lett.'

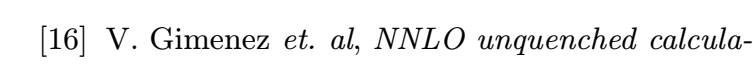
L - - - - tion of the_b quark mass, hep-

[17] P. Ball, M. Beneke and V. Braun, 'Phys. Rev.' (195) 3929

[18] C.A. Dominguez, in Proc. of the Third Workshop on the Tau-Charm Factory, Marbella,

- - - - Spain_(1993), Ed_ J_Kirkby-and_R Kirkby, Edi' - - - - tions-Erontiéres, p_357. B 301 (1993) 101'; 'Phys. Rev. D 47 (1993)'

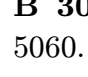

[39] M. Huang, C. Li and Y. Dai, QCD sum rule analysis of the subleading Isgur-Wise formfactor $\tau_{1}\left(v v^{\prime}\right)$ and $\tau_{2}\left(v v^{\prime}\right)$ for $B \rightarrow D_{1} \ell \bar{\nu}$ and $B \rightarrow D_{2}^{*} \ell \bar{\nu}$, hep-ph/9909307!

[40] T.M. Aliev, V.L. Eletsky and Ya.I. Kogan, 'Sov.' J. Nucl. Phys. 40 (1984) 527.

[19] The BaBar Physics Book, Appendix D, Ed.: P.F. Harrison and H.R. Quinn, SLAC-R-504 (1998).

[41] T.M. Aliev, A.A. Ovchinnikov and V.A. Slobodenyuk, Preprint IC/89/382 (unpublished).

[42] P. Ball et al., 'Phys. Lett. B 259 (1991) 481', 
[43] P. Ball, V.M. Braun and H.G. Dosch, P'Phys.' - _ - Rev. D $44(1991) 3567$.

[44] P. Ball, 'Phys. Rev. D_48 (1993)_3190!

[45] A. Khodjamirian, R. Rückl, S. Weinzierl, C.W. Winhart and O. Yakovlev, Predictions on $B \rightarrow \pi \bar{l} \nu, D \rightarrow \pi \bar{l} \nu$ and $D \rightarrow K \bar{l} \nu$ from QCD Light-Cone Sum Rules, Preprint WUEITP-99-017, paper in preparation.

[46] A. Khodjamirian, R. Ruckl and C.W. Winhart,

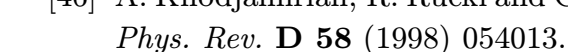

L - . - L

[47] I.I. Balitsky, V.M. Braun and A.V. Kolesnichenko, Sov. J. Nucl. Phys. 44 (1986) 1028; I-ン--

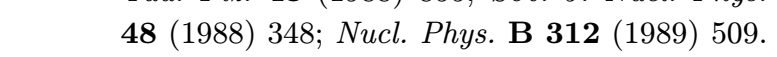
$=-[48]$ V $M$ M.Braun and I.E. Filyanov, ᄂ = : = =

[49] V.L. Chernyak and I.R. Zhitnitsky, iNucl. Phys.' '- - - B $345(1990)-137$

[50] V.M. Braun, Light cone sum rules, hep-ph/9801222!

[51] A. Khodjamirian and R. Rückl, QCD sum rules_for exclusive decays of heavy mesons,

1. - - hep-ph/9801443!

[52] P. Ball and V.M. Braun, 'Phys. Rev. D 55 [ _ .

53] P. Ball, V.M. Braun and H.G. Dosch iPhys [ - . Lett. B 273. (1991) 316.

[54] S. Narison, 'Phys. Lett. B $\mathbf{2 8 3}(1992) 384_{1}^{\prime}$ [55] P. Colangelo and P. Santorelli, Phys. Lett. Bi $\mathbf{3 2 7}(1994) 123$

[56] V.M. Belyaev, A. Khodjamirian and R. Rückl, Z. Physik C $\mathbf{6 0}(1993) 349$

[57] A. Khodjamirian et al., 'Phys. Lett. B 410!'

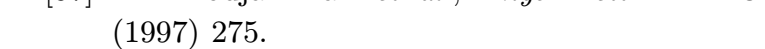

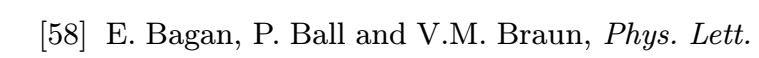
(1)

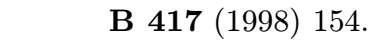

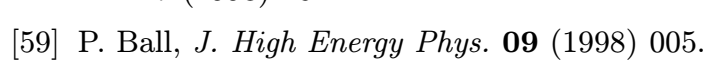

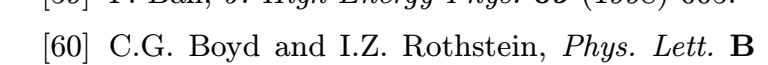

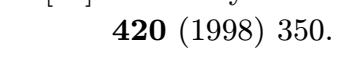

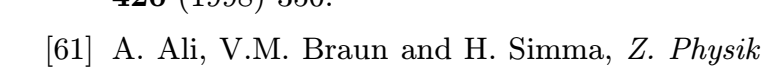

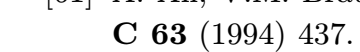

[62] T.M. Aliev, A. Ozpineci and M. Savci, ṔPhys.' Rev. $\mathbf{D} \mathbf{5 6}(1997) 4260$.

[63] P. Ball and V.M. Braun, 'Phys. Rev. D $\mathbf{5 8}$ i -

[64] P. Ball et al. 'Nucl. Phys. B 529 (1998) 323i P. Ball and V.M Braun, iNucl. Phys. B 543i (1999) 201
[65] D. Becirevic and A.B. Kaidalov, Comment on the heavy $\rightarrow$ light form-factors, hep-ph/9904490'.

[66] UKQCD Collaboration, L. Del Debbio et al., 'Phys. Lett. B_416 (1998)_392.'.

[67] A. Ali et al., A Comparative study of the decays $B \rightarrow(K, K *) \ell^{+} \ell^{-}$in standard model and supersymmetric theories, hep-ph/9910221.'.

[68] J. Charles et al., 'Phys. Rev. D $\mathbf{6 0}(1999) !$ 014001 .'.

[69] T.M. Aliev et al., Phys. Rev. D $\mathbf{5 3}(1996) 355$

[70] T.M. Aliev, E. Iltan and N.K. Pak, 'Z. Physih C 73 (1997) 293'.

[71] T.M. Aliev, N.K. Pak and M. Savci, iPhys. Lett.' B 390 (1997) 335.

[72] P. Colangelo and F. De Fazio, Eur.Phys.J. C4 (1998) 503.

[73] A. Khodjamirian and R. Rückl, Exclusive Nonleptonic Decays of Heavy Mesons in QCD, hep-ph/9807495.

[74] B.Yu. Blok and M.A. Shifman, 'Sov. J. Nucl.' Phys. 45 (1987) 135,301,522.

[75]_A. Khodjamirian, G. Stoll and D. Wyler, Phys.' Lett. B 358 (1995) 129".

[76] A. Ali and V.M. Braun, IPhys. Lett. B $\mathbf{3 5 9}$ (1995) 223.

[77] A. Khodjamirian et al., 'Phys. Lett. B _ 402' $(1997)$ 167.

[78] P. Colangelo and F. De Fazio, 'Phys. Lett. B! $\mathbf{3 8 7}(1996) 371$.

[79] Y.-B. Dai et al., 'Phys. Lett. B $\mathbf{3 8 7}(1996)$ 379'.

[80] C.-S. Huang, C.-F. Qiao and H.-G. Yan, 'Phys.। Lett. B $43 \overline{7}(1998) 403$ '

[81] R.S.M. de Carvalho et al., 'Phys. Rev. D $\mathbf{6}$ ' $(1 \overline{9} 99) 03 \overline{4009}$

[82] C.-S. Huang and H.-G. Yan, 'Phys. Rev. D_59' $(1999)$ 114022,

[83] A.G. Grozin and O.I. Yakovlev, 'Phys. Lett.' B 285 (1992) 254; see an update in hep-ph/9908364'.

[84] O.I. Yakovlev, Heavy_baryons and QCD sum rules, 'hep-ph/9608348;

[85] V.V. Kiselev, A.A. Likhoded and A.I. Onishchenko, Semileptonic $B_{c}$ meson decays in sum rules of $Q C D$ and NRQCD, hep-ph/9905359. 